\title{
Artificial Neural Network Based System Identification for a Single-Shaft Gas Turbine
}

\author{
Hamid Asgari, ASME Member, XiaoQi Chen, ASME Member, Mohammad B. Menhaj, and Raazesh Sainudiin
}

\begin{abstract}
During recent decades, artificial intelligence has been employed as a powerful tool for identification of complex industrial systems with nonlinear dynamics, such as gas turbines (GT). In this study, a methodology based on artificial neural network (ANN) techniques was developed for offline system identification of a low-power gas turbine. The processed data was obtained from a SIMULINK model of a gas turbine in MATLAB environment. A comprehensive computer program code was generated and run in MATLAB environment for creating and training different ANN models with two-layer feed-forward multi-layer perceptron (MLP) structure. The code consisted of various training functions, different number of neurons as well as a variety of transfer (activation) functions for hidden and output layers of the network. It was shown that the optimal model for a two-layer network with MLP structure, consisted of 20 neurons in its hidden layer and used trainlm as its training function, as well as tansig and logsid as its transfer functions for the hidden and output layers. It was also observed that trainlm has a superior performance in terms of minimum MSE, compared with each of the other training functions. The resulting model could predict performance of the system with high accuracy. The methodology provides a comprehensive view of the performance of over 18720 ANN models for system identification of single shaft GT. One can use the optimal ANN model from this study when training from real data obtained from this type of GT. This is particularly useful when real data is only available over a limited operational range.
\end{abstract}

Index Terms-Gas turbine, system identification, modelling, simulation, optimization, neural network.

\section{INTRODUCTION}

$\mathrm{G}$ AS turbines have been used widely in industrial plants all $\mathbf{J}_{\text {over the world, and specifically in places such as offshore }}$ plants and oil fields which are far away from urban areas. GTs are the main source of power generation. Their key role in this developing industry has motivated researchers to explore new

Manuscript received February 23, 2013. This work was supported by Premier Scholarship, Department of Mechanical Engineering at University of Canterbury (UC), Private Bag 4800, Christchurch 8140, New Zealand.

Hamid Asgari is with the Department of Mechanical Engineering at UC (email: hamid.asgari@pg.canterbury.ac.nz and/or asgari_ha@yahoo.com).

XiaoQi Chen is with the Department of Mechanical Engineering at UC (email: xiaoqi.chen@canterbury.ac.nz).

Mohammad Bagher Menhaj is with the Department of Electrical Engineering at Amir Kabir University of Technology (AUT), Tehran, Iran (email: menhaj@aut.ac.ir).

Raazesh Sainudiin is with the Department of Mathematics and Statistics at UC (e-mail: r.sainudiin@math.canterbury.ac.nz). methods to predict dynamic behaviour of these complex systems as accurately as possible. A variety of analytical and experimental techniques has been developed so far to approach an optimal model of gas turbines. Artificial neural network is one of the techniques that has been playing a significant role in system identification and modelling of industrial systems during recent decades. Although using artificial neural network for industrial applications is still a controversial issue, its capability to capture dynamics of the systems without any prior knowledge about them and their complicated dynamical equations, is an important advantage. There are considerable research activities in the field of ANNbased system identification and modelling of gas turbines. In spite of considerable research activities that have been carried out so far in system identification and modelling of gas turbines using artificial neural networks, system optimization is still a challenging and controversial issue. Because of sophisticated and nonlinear dynamic behaviour of GTs, significant effort and attention still needs to be paid to the dynamics of these systems. These efforts aim to unfold unknowns behind undesirable events such as unpredictable shutdowns, over-heating and over-speed during gas turbine operation. Fortunately, using black-box system identification and modelling techniques such as ANN, can effectively assist researchers who work in this area. The study in this area can be categorised into aero gas turbine models and stationary (mainly power plant) gas turbine models [2].

In the area of aero gas turbine models, one can refer to the efforts of Chiras et al. [3]-[6], Ruano et al. [7], and Torella et al. [8]. They employed a variety of ANN-based techniques and approaches such as MLP, nonlinear auto-regressive moving average with exogeneous inputs (NARMAX), nonlinear autoregressive exogenous model (NARX), radial basis function (RBS), back propagation neural networks (BPNN) and Bspline, to explore nonlinear dynamics of aero gas turbines.

Some ANN-based research activities were concentrated on power plant gas turbines. The major contributions in this area included the studies carried out by Lazzaretto and Toffolo [9], Jurado [10], Bartolini et al. [11], Bettocchi et al. [12], [13], Basso et al. [14], Yoru et al. [15], Simani et al., [16], Fast et al. [17]-[22], Spina et al. [23], Ogaji et al. [24], and Arriagada et al. [25]. The results of these studies have indicated that ANN can be very useful for the real time simulation of gas turbines, especifically when there is not enough information about the system dynamics [12]. It was also shown that ANN can be used as a high-speed powerful tool for real-time control 
problems [24]. ANN has the capability to identify system faults and to generate warnings at early stages with high reliability [25].

As it can be seen from the literature survey, each of the research activities in the field of ANN based gas turbine system identification, investigated the issue from a specific perspective and has its own limitation(s). For instance, Chiras et al. [3]-[6], Ruano et al. [7], and Torella et al. [8], concentrated on aero gas turbines. Jurado [10], and Bartolini et al. [11], investigated micro gas turbines. Ogaji et al. [24], Arriagada et al. [25], and Fast et al. [19]-[22], explored applications of ANN for fault diagnosis, condition monitoring and/or sensor validation purposes. Fast et al. [18] just considered the full load situation for ANN-based system identification and modelling single-shaft gas turbines. In some GT models, the nonlinear terms in the model were restricted to the second order [10]. Besides, most of the ANN based models of gas turbines were built on the basis of a specific training function ('trainlm') and transfer functions ('tansig' or 'logsig' type in the hidden layer, and 'purelin' type in the output layer).

According to the methodology used in this study, various backpropagation training functions, different number of neurons and a variety of transfer functions were employed to train the network in order to explore an optimal ANN model using MLP structure. To increase the level of generalisation for the model, the data sets were partitioned randomly for training, validation and test purposes.

In this study, firstly a brief description of gas turbine performance is presented. Then, a rebuilt SIMULINK model of a low power gas turbine based on a previous research by Ailer et al. [1] is briefly presented. The next section of the paper describes ANN based system identification processes including generating the required data sets for training of the network using the SIMULINK model, writing the computer code and training the network. Finally, the results of the study are presented and concluding remarks are discussed.

\section{GAS TURBINE PERFORMANCE}

Gas turbine is considered as an internal combustion engine which uses the gaseous energy of air to convert chemical energy of fuel to mechanical energy. Figure 1 shows a schematic of the main components of a single-shaft gas turbine engine; including compressor, combustion chamber (combustor), and turbine. The set of these components is called engine core or gas generator (GG). Compressor and turbine are connected by the central shaft and rotate together. As the figure shows, air enters the compressor at section 1 and is compressed through passing the compressor. The hot and compressed air enters the combustion chamber (combustor) at section 2. In combustor, fuel is mixed with air and ignited. The hot gases which are the product of combustion are forced into the turbine at section 3 and rotate it. Turbine drives the compressor and the GG mechanical output, which can be a large pump, a large compressor or an electricity generator in a power plant station.
Gas turbines work based on Brayton cycle. Figure 2 shows a typical standard Brayton cycle in temperature-entropy frame [26]. As it can be seen from the figure, the actual processes in the compressor (1-2) and turbine (3-4) are irreversible and non-isentropic. Points 2s and 4s show the ideal situation, when these processes are assumed isentropic. Neglecting pressure loss in the air filters and the combustion chamber, processes 23 and 4-1 can be considered isobar [26].

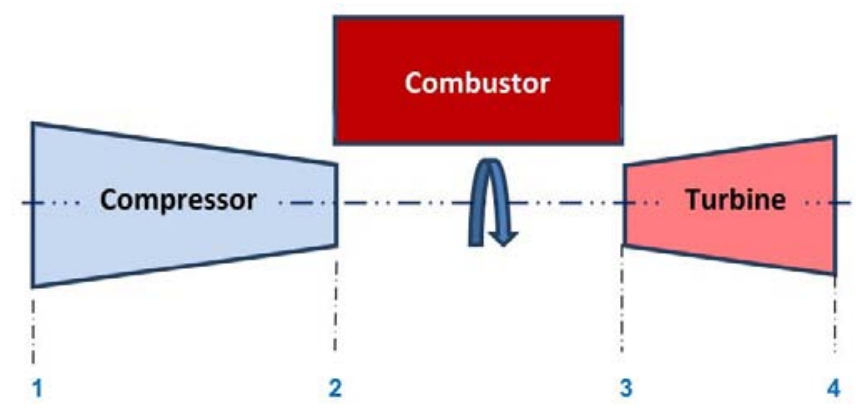

Fig. 1. A schematic of a typical single-shaft gas turbine.

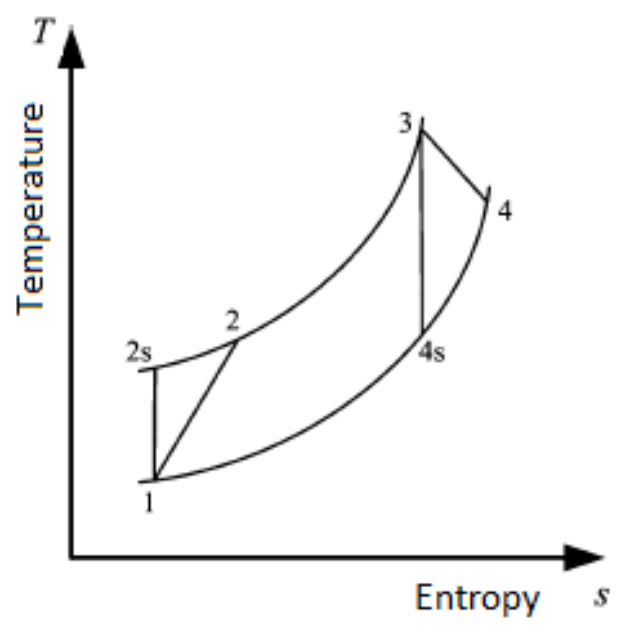

Fig. 2. Typical Brayton cycle in temperature-entropy frame [26].

\section{GAS TURBINE SIMULINK MODEL}

The data for this study was generated using a re-simulated nonlinear dynamic model of a low-power single shaft gas turbine. The model has been already developed and verified for loop-shaping control purposes by Ailer et al. [1]. The main idea behind the study was to improve dynamic response of the engine by implementation of a developed nonlinear controller. The model was developed and simulated in SIMULINK/MATLAB, based on engineering principles, gas turbine dynamics, constitutive algebraic equations, and by using operational data. Model verification was performed by 
open-loop simulations against qualitative operation experience and engineering intuition [1].

In this study, the SIMULINK model was rebuilt using the same principles and equations. The equations 1,2 and 3 , indicate the main equations of the gas turbine employed in the SIMULINK model [1]. Definition of each of the parameters in these equations is provided in Table I.

TABLE I

DEFINITION OF PARAMETERS

\begin{tabular}{|c|c|c|}
\hline Parameter & Symbol & Unit \\
\hline rotational speed (number of revolutions) & $n$ & $1 / \mathrm{Sec}$ \\
\hline temperature at section 1 & $T_{01}$ & $K$ \\
\hline temperature at section 2 & $T_{02}$ & $K$ \\
\hline temperature at section 3 & $T_{03}$ & $K$ \\
\hline temperature at section 4 & $T_{04}$ & $K$ \\
\hline pressure at section 1 & $P_{01}$ & $\mathrm{~Pa}$ \\
\hline pressure at section 2 & $P_{02}$ & $P a$ \\
\hline pressure at section 3 & $P_{03}$ & $P a$ \\
\hline pressure at section 4 & $P_{04}$ & $\mathrm{~Pa}$ \\
\hline air mass flow rate in compressor & $\dot{m}_{C}$ & $\mathrm{Kg} / \mathrm{Sec}$. \\
\hline gas mass flow rate in turbine & $\dot{m}_{T}$ & $\mathrm{Kg} / \mathrm{Sec}$ \\
\hline fuel mass flow rate & $\dot{m}_{\text {fuel }}$ & $\mathrm{Kg} / \mathrm{Sec}$. \\
\hline gas mass in combustion chamber & $m_{\text {comb }}$ & $K g$ \\
\hline time & $t$ & Sec. \\
\hline specific heat of air in constant pressure & $C_{P_{\text {air }}}$ & $J / k g K$ \\
\hline specific heat of gas in constant pressure & $C_{P_{\text {gas }}}$ & $\mathrm{J} / \mathrm{kg} \mathrm{K}$ \\
\hline medium Specific heat in constant volume & $C_{v_{\text {med }}}$ & $\mathrm{J} / \mathrm{kg} \mathrm{K}$ \\
\hline fuel lower thermal value & $Q_{f}$ & $\mathrm{~J} / \mathrm{kg}$ \\
\hline combustion chamber efficiency & $\eta_{\text {comb }}$ & - \\
\hline mechanical efficiency & $\eta_{\text {mech }}$ & - \\
\hline inertial moment & $\Theta$ & Kg. $\mathrm{m}^{2}$ \\
\hline moment of load & $M_{\text {load }}$ & N.m \\
\hline
\end{tabular}

$$
\begin{aligned}
& \frac{d m_{\text {comb }}}{d t}=\dot{m}_{C}+\dot{m}_{f u e l}-\dot{m}_{T} \\
& \begin{aligned}
\frac{d P_{03}}{d t}=\frac{P_{03}}{m_{c o m b}} & \left(\dot{m}_{C}+\dot{m}_{f u e l}-\dot{m}_{T}\right) \\
& +\frac{P_{03}}{T_{03} C_{\text {vmed }} m_{\text {comb }}}\left(\dot{m}_{c} C_{p_{\text {air }}} T_{02}\right. \\
& -\dot{m}_{T} C_{p_{\text {gas }}} T_{03}+Q_{f} \eta_{\text {comb }} \dot{m}_{f u e l} \\
& \left.-C_{\text {vmed }} T_{03}\left(\dot{m}_{C}+\dot{m}_{f u e l}-\dot{m}_{T}\right)\right)
\end{aligned}
\end{aligned}
$$

$$
\begin{aligned}
\frac{d n}{d t}=\frac{1}{4 \pi^{2} \ominus n} & \left(\dot{m}_{T} C_{p_{\text {gas }}}\left(T_{03}-T_{04}\right) \eta_{\text {mech }}\right. \\
& -\dot{m}_{c} C_{p_{\text {air }}}\left(T_{02}-T_{01}\right) \\
& \left.-2 \pi \frac{3}{50} n M_{\text {load }}\right)
\end{aligned}
$$

Figure 3 shows a simplified SIMULINK model of the gas turbine plant. $\dot{m}_{\text {fuel }}, M_{\text {load }}, T_{01}$, and $P_{01}$ were considered as inputs of the model. The other GT parameters can be considered as outputs of the system. In figure $3, n$ and $T_{04}$ are shown as the outputs of the system.

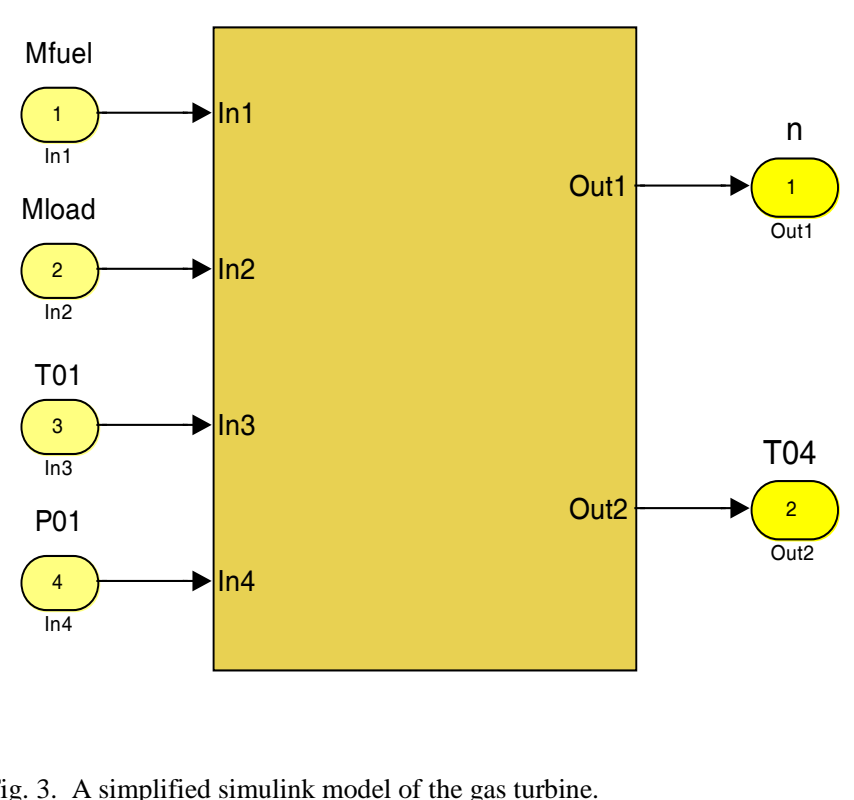

\section{ANN-BASED SYSTEM IDENTIFICATION}

Artificial neural network is a data-driven model. It has been considered as a suitable alternative to white-box models during the last few decades. ANN is a group of interconnected artificial units (neurons) with linear or nonlinear transfer functions. Neurons are arranged in different layers including input layer, hidden layer(s) and output layer. The number of neurons and layers in an ANN model depends on the degree of complexity of the system dynamics. ANN learns the relation between inputs and outputs of the system through an iterative process called training. Each input into the neuron has its own associated weight. Weights are adjustable numbers which are determined during training the network. ANN-based system identification for the gas turbine was carried out during data generation and training processes. 


\section{A. Data Generation}

ANN-based models can be created directly from the operational data from an actual gas turbine, or simulated data from original equipment manufacturers (OEMs) performance. Simulated data may be used when operational data are not available. The obtained data should cover the whole operational range of the system. All transient data during start or stop process should be removed from the collected data before the modelling process. Selecting the right parameters of GAS TURBINEs as inputs and outputs of the neural network is very important for making an accurate and reliable model. The availability of data for the selected parameters, system knowledge for identification of interconnections between different parameters and the objectives for making a model are basic factors in choosing appropriate inputs and outputs.

In this study, about 3000 data sets were generated for the whole operational range of the engine using the SIMULNK model that has already been developed by Ailer [1]. These data sets were employed for training an optimized ANN model for the purpose of system identification. In this work, four independent variables including fuel rate, ambient temperature, ambient pressure and load, were considered as inputs. The outputs of the ANN model consisted of seventeen dependent parameters. Tables 2 and 3 show the inputs and outputs(targets) of the model. Indices 1, 2, 3 and 4 refer to the corresponding sections in figure 1.

\section{B. Training Process}

During the process of creating ANN models for gas turbines, a variety of approaches can be applied based on the flexibility that ANN provides. This flexibility is due to different factors including number of neurons, type of transfer functions, and type of training algorithm. It has been already shown that any muti-dimentional nonlinear mapping of any continuous function can be carried out by a two-layer MLP with suitable chosen number of neurons in its hidden layer [27]. In this work, an ANN model with a two-layer feedforward MLP structure was employed for system identification. Figure 4 shows a schematic of the ANN structure of the gas turbine. As it can be seen from the figure, the inputs correspond to the four independent GT parameters and the desired outputs including seventeen different dependent GT parameters. The ANN can be named 4-H-17 according to its structure with one hidden layer.

TABLE II

INPUT PARAMETERS

\begin{tabular}{lccc}
\hline \hline Parameter & Symbol & Unit & Operational Range \\
\hline fuel rate & $M_{f}$ & $K g / S e c$. & {$[0.00367 ; 0.027]$} \\
Ambient & $T_{01}$ & $K$ & {$[243.15 ; 308.15]$} \\
temperature & & $K P a$ & {$[60 ; 110]$} \\
ambient pressure & $P_{01}$ & $N . m$. & {$[0 ; 363]$} \\
\hline \hline
\end{tabular}

TABLE III OUTPUT PARAMETERS

\begin{tabular}{lcc}
\hline \hline Parameter & Symbol & Unit \\
\hline rotational speed (number of revolutions) & $N$ & $1 / \mathrm{Sec}$. \\
temperature at section 1 & $T_{01}$ & $\mathrm{~K}$ \\
temperature at section 2 & $T_{02}$ & $\mathrm{~K}$ \\
temperature at section 3 & $T_{03}$ & $\mathrm{~K}$ \\
temperature at section 4 & $T_{04}$ & $\mathrm{~K}$ \\
pressure at section 1 & $P_{01}$ & $\mathrm{KPa}$ \\
pressure at section 2 & $P_{02}$ & $\mathrm{KPa}$ \\
pressure at section 3 & $P_{03}$ & $\mathrm{KPa}$ \\
pressure at section 4 & $P_{04}$ & $\mathrm{KPa}$ \\
mass flow rate & $M_{a}$ & $\mathrm{Kg} / \mathrm{Sec}$. \\
compressor efficiency & $\eta_{C}$ & - \\
turbine efficiency & $\eta_{T}$ & - \\
compressor work & $W_{C}$ & $\mathrm{Kw}$ \\
turbine work & $W_{T}$ & $\mathrm{Kw}$ \\
net work & $W_{n e t}$ & $\mathrm{Kw}$ \\
gas turbine efficiency & $\eta_{G T}$ & - \\
specific fuel consumption & $S F C$ & $\mathrm{Kg} / \mathrm{KwH}$ \\
fuel to air mass flow rates ratio & $F$ & - \\
compression ratio in compressor & $R_{c p}$ & - \\
\hline \hline
\end{tabular}

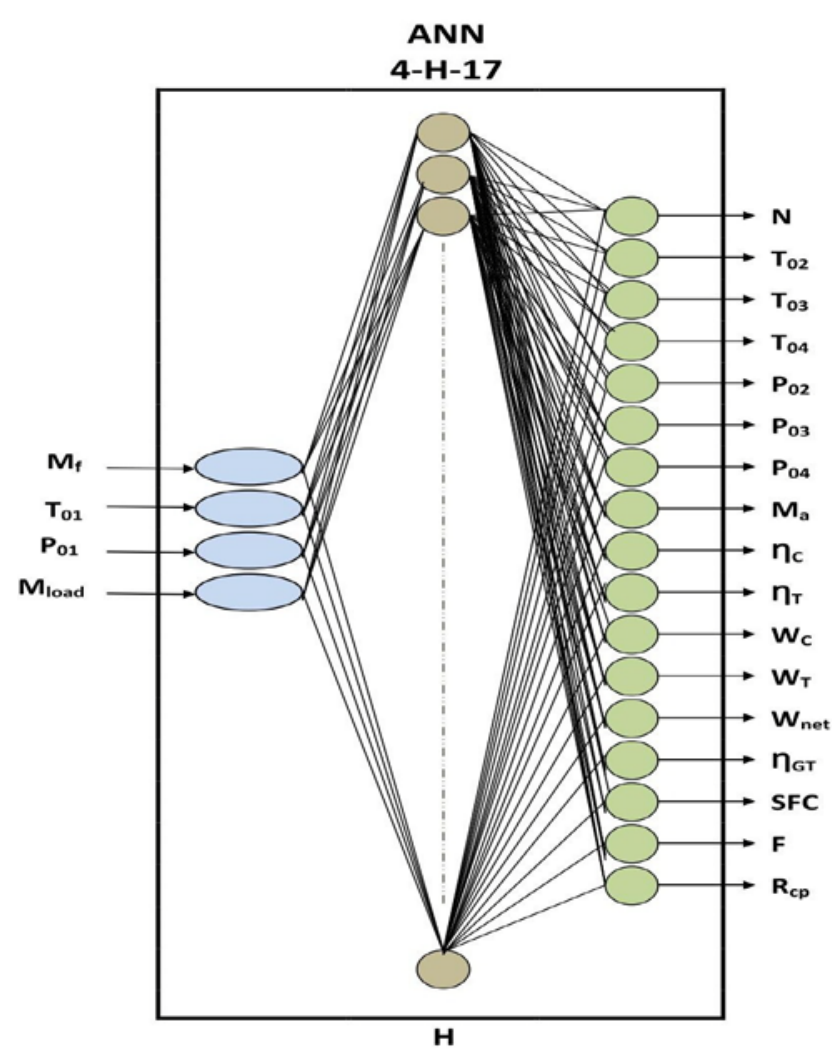

Fig. 4. A schematic of the ANN structure for the gas turbine engine. 


\section{Code Generation}

To obtain an optimized network structure and to assure good generalisation characteristic of the gas turbine model, a comprehensive computer code was generated and run in MATLAB for a two-layer MLP network consisting of various backpropagation training functions, transfer functions and different number of neurons. The thirteen different training functions, applied in the code, included trainbfg, trainb, traincgb, traincgf , traincgp , traingd , traingda, traingdm , traingdx, trainlm, trainoss, trainrp, and trainscg. Six transfer functions consisted of tansig, logsig, purelin, hardlim, satlin, and poslin. The number of neurons tried the program was from 1 to 40 . Figure 5 shows the flow diagram of the computer code for ANN-based system identification of the gas turbine.

As it can be seen from figure 5, after feeding and normalizing the data sets, they are randomly partitioned into training (70\%), validation (15\%) and test (15\%) categories. At the next step, the structure of the network (MLP) is specified. After determination of number of neurons in the hidden layer, training function as well as transfer functions for the hidden and output layers, the training process of the network is started and repeated two more times for the same adjusted factors, so that the best performance among the three trials, is specified and recorded in a matrix. The process is repeated in four main loops of the code for different number of neurons (1 to 40), different types of backpropagation training functions, and combination of different transfer functions for the hidden and output layers. The results of the all performances are recorded into a matrix and are sorted on the basis of their performance measure (MSE). According to the code, for each training process, the best performance is calculated as an average of the best test performance and one-epoch-ahead of the best validation performance, which is called average performance in this paper. Epoch is a neural network term for iteration in a training process. The number of epochs shows the number of times that all the patterns are presented to the neural network. More epochs means more training time. In each epoch of an ANN, all the weight values of the neurons are updated. In this study, one thousand epochs was considered for the whole training process of the ANN, to be sure that the training would not be stopped before reaching a dominating local minimum. The optimal ANN model was recognized from the sorted results and tested again for final verification.

\section{RESULTS}

In order to find the best model for the gas turbine engine, the generated code was run in MATLAB and 18720 $(40 * 13 * 6 * 6)$ different ANN structures were trained using randomly partitioned data sets for training, validation and test purposes. The results of the trainings were recorded and the performance was evaluated and compared in terms of their mean squared error (MSE). Finally, an optimal ANN with minimum MSE was selected and tested again to assure good generalization characteristics of the model. The results from the model for different parameters of the gas turbine (predicted values) were compared with the values of the generated data from the SIMULINK model.

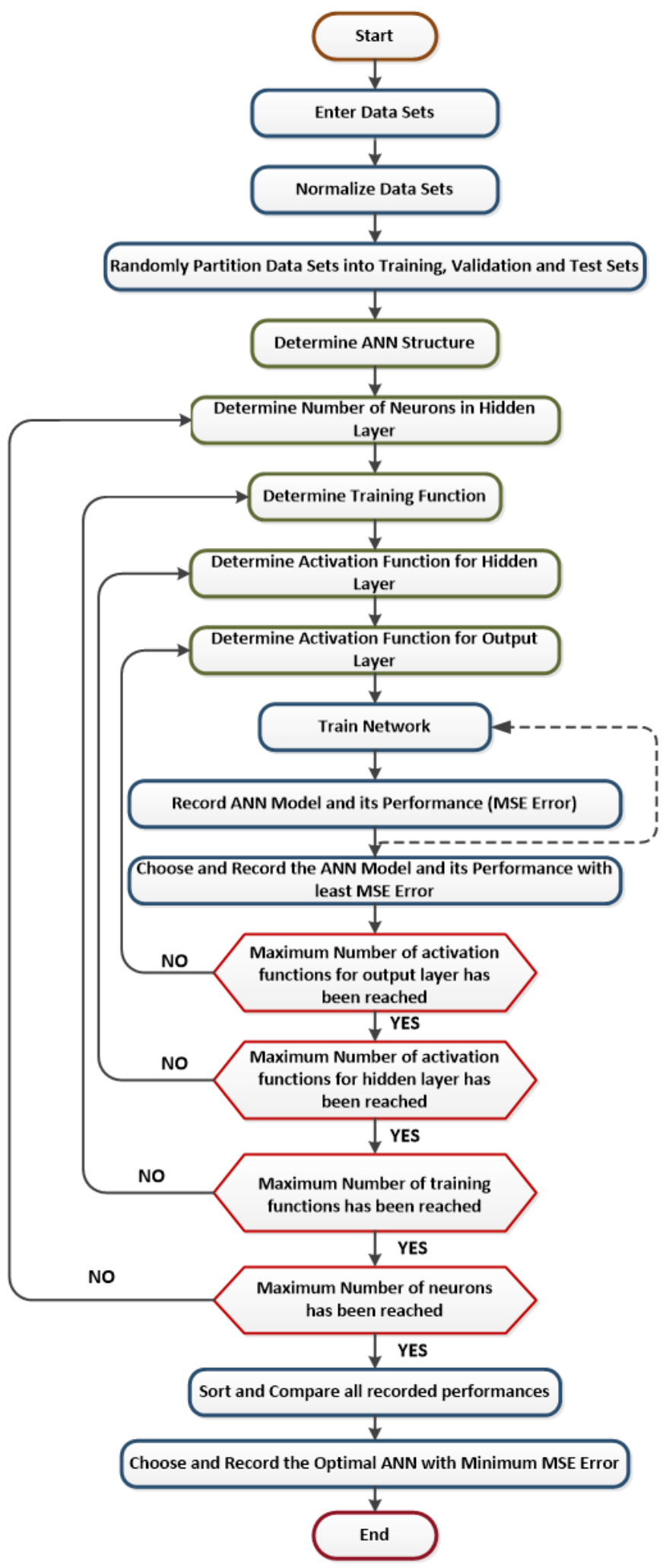

Fig. 5. Flow diagram of the generated computer code for ANN-based system identification of the gas turbine. 
Table IV indicates the best performance in terms of different training functions. As it can be seen, a two-layer MLP using training function trainlm, transfer functions tansig and logsig for the hidden and output layers, with 20 neurons showed the best performance (the least MSE). It can also be seen that 'trainlm' has a superior performance in terms of minimum MSE, compared with each of the other training functions.

Figure 6 shows details of the most optimal trained network based on the average performance of all the trained structures. Performance of the ANN for training, validation, and test has been also shown in the figure. As it can be seen, the iteration in which the validation performance error reached the minimum was 24 . The mean square error of the performance at this point was quite low. The training continued for 10 more iteration before the training stopped.

Figure 7 shows the related regression plot, which indicates the relationship between the outputs of the network and the outputs of the system (targets). The $\mathrm{R}$ value is an indication of the relationship between the outputs and the targets. As the figure shows, R values for the all graphs are very close to 1 . Therefore, the result for each of training, validation, and testing data sets indicates a very good fit.

TABLE IV

BEST PERFORMANCE FOR DIFFERENT TRAINING FUNCTIONS

\begin{tabular}{|c|c|c|c|c|}
\hline $\begin{array}{l}\text { Training } \\
\text { Function }\end{array}$ & $\begin{array}{l}\text { Number } \\
\text { of } \\
\text { Neurons }\end{array}$ & $\begin{array}{l}\text { Transfer } \\
\text { Function in } \\
\text { Hidden } \\
\text { Layer }\end{array}$ & $\begin{array}{c}\text { Transfer } \\
\text { Function in } \\
\text { Output } \\
\text { Layer }\end{array}$ & $\begin{array}{l}\text { Best Average } \\
\text { Performance } \\
\text { (MSE) }\end{array}$ \\
\hline trainlm & 20 & tansig & logsig & 2.49E-06 \\
\hline traincgp & 20 & purelin & hardlim & $1.11 \mathrm{E}-05$ \\
\hline traincgf & 20 & logsig & logsig & $1.15 \mathrm{E}-05$ \\
\hline traincgb & 18 & logsig & satlin & 1.16E-05 \\
\hline trainscg & 20 & tansig & satlin & $1.36 \mathrm{E}-05$ \\
\hline trainbfg & 19 & purelin & satlin & $1.50 \mathrm{E}-05$ \\
\hline trainoss & 20 & tansig & logsig & $6.68 \mathrm{E}-05$ \\
\hline trainrp & 16 & tansig & satlin & 0.000189 \\
\hline traingdx & 15 & tansig & satlin & 0.000251 \\
\hline traingda & 10 & satlin & satlin & 0.000688 \\
\hline trainb & 4 & hardlim & hardlim & 0.063675 \\
\hline traingdm & 2 & tansig & logsig & 0.087112 \\
\hline traingd & 2 & logsig & hardlim & 0.090159 \\
\hline
\end{tabular}

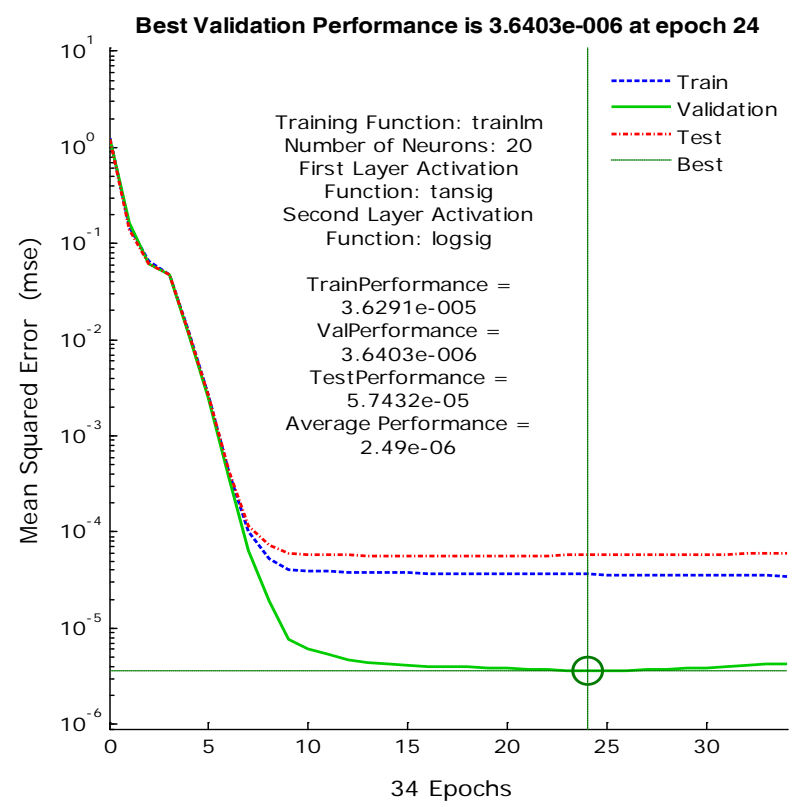

Fig. 6. Performance of the optimal ANN.
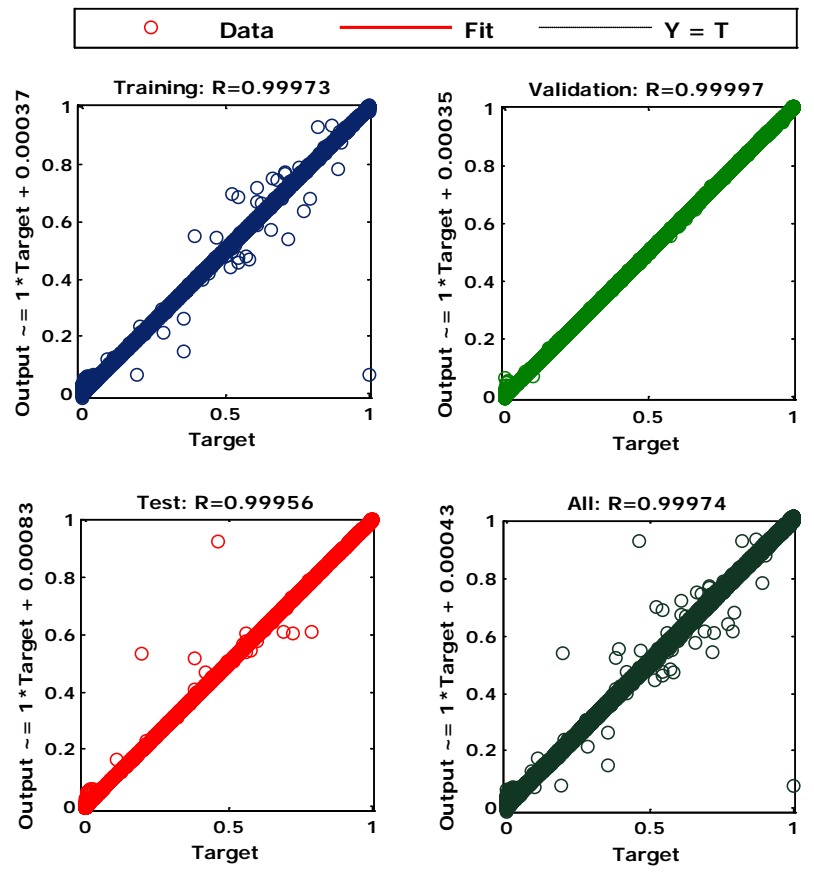

Fig. 7. Regression of the optimal ANN.

Figures 8 and 9 compare actual and predicted data for rotational speed and temperatures in different sections of the gas turbine respectively. Actual data or targets are the data obtained from the SIMULINK model. Predicted data are outputs of the ANN model. For clarity of the figures, just outputs of 100 data sets have been shown. As the figures show, the outputs of the ANN model followed the targets precisely. It shows that the resulted ANN model can predict the reaction of the system to changes in independent parameters with high accuracy and is capable of system identification with high reliability. 


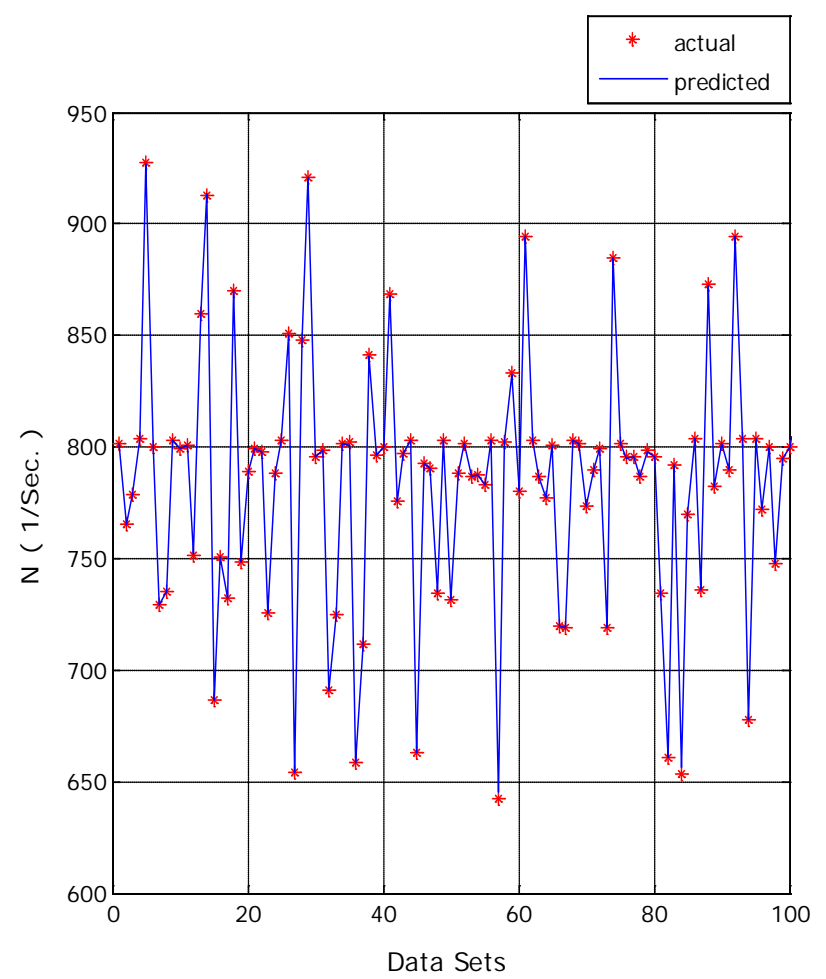

Fig. 8. Comparison between outputs of the SIMULINK model and the optimal ANN model for gas turbine rotational speed.
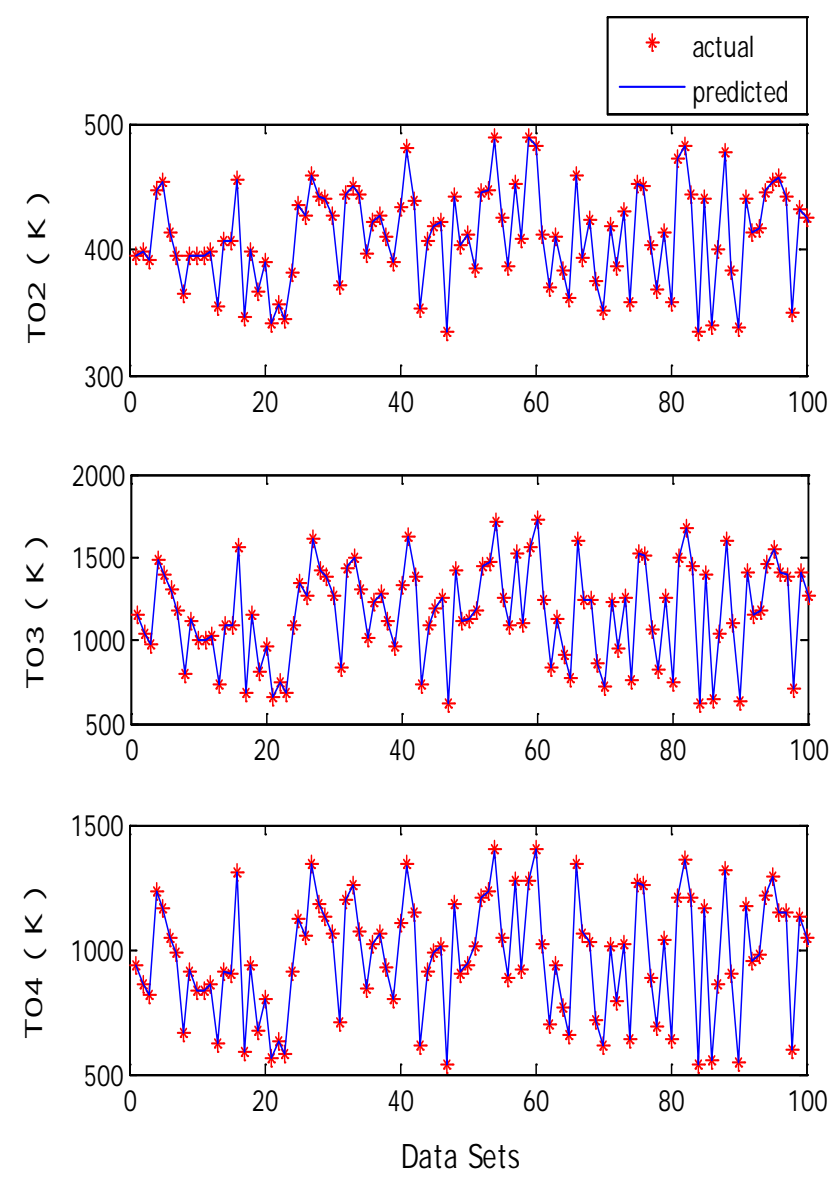

Fig. 9. Comparison between outputs of the SIMULINK model and ANN model for gas turbine temperatures.

\section{CONCLUSION}

Artificial neural network has been used as a robust and reliable technique for system identification and modeling of complex systems with nonlinear dynamics such as gas turbines. It can provide outstanding solutions to the problems that cannot be solved by conventional mathematical methods. However, ANN-based techniques, in their own turn, can be applied to the systems through a variety of approaches which include different structures and training methods. In this study, a new ANN-based methodology was developed for offline system identification of a low-power gas turbine. A comprehensive computer program code was generated and run in MATLAB environment using the obtained data from a resimulated model of a gas turbine in SIMULINK. Code generation was on the base of combinations of various training functions, number of neurons and transfer functions for ANN with two-layer MLP structure. The resulting model showed that the ANN-based method can be applied reliably for system identification of gas turbines. It can precisely predict dependent parameters of the gas turbine based on the changes in the independent inputs of the system. The methodology of this study can be used to predict performance of similar gas turbine systems with high accuracy. The upcoming studies in this field can lead to design and manufacturing of gas turbines with optimal performance and minimal supervision and energy consumption.

\section{REFERENCES}

[1]. P. Ailer, I. Santa, G. Szederkenyi, and K.M. Hangos, "Nonlinear modelbuilding of a low-power gas turbine, "PERIODICA POLYTECHNICA TRANSPORTATION ENGINEERING, 29(1-2):117_135, 2002.

[2]. H. Asgari, X.Q. Chen, M.B. Menhaj, and R. Sainudiin, "ANN-based system identification, modelling and control of gas turbines - A review," Advanced Materials Research, 622-623, 611, 2012

[3]. N. Chiras, C. Evans, and D. Rees, "Nonlinear gas turbine modelling using NARMAX structures," IEEE Transactions on Instrumentation and Measurement, VOL.50, NO.4: 893-898, 2001.

[4]. N. Chiras, C. Evans, and D. Rees, "Nonlinear modelling and validation of an aircraft gas turbine engine," School of Electronics, University of Glamorgan, Wales, UK, 2001.

[5]. N. Chiras, C. Evans, and D. Rees, " Nonlinear gas turbine modelling using feedforward neural networks,” University of Glamorgan, School of Electronics, Wales, UK, 2002.

[6]. N. Chiras, C. Evans, and D. Rees, "Global nonlinear modelling of gas turbine dynamics using NARMAX structures,” Journal of Engineering for Gas Turbines and Power, Vol. 124 / 817, 2002.

[7]. A.E. Ruanoa, P.J. Fleming, C. Teixeiraa, K.R. Vazquezc, and C.M. Fonsecaa, "Nonlinear identification of aircraft gas turbine dynamics," Neuro Computing 55: 551 - 579, 2003.

[8]. G. Torella, F. Gamma, and G. Palmesano, "Neural networks for the study of gas turbine engines air system," Proceedings of the International Gas Turbine Congress, Tokyo, Japan, 2003.

[9]. A. Lazzaretto, and A. Toffolo, "Analytical and neural network models for gas turbine design and off-design simulation.” International Journal of Applied Thermodynamics, Vol.4, No.4: 173-182, 2001.

[10]. F. Jurado, "Nonlinear modelling of micro-turbines using NARX structures on the distribution feeder," Energy Conversion and Management 46: 385-401, 2005.

[11]. C.M. Bartolini, F. Caresana, G. Comodi, L. Pelagalli, M. Renzi, and S. Vagni, "Application of artificial neural networks to micro gas turbines," Energy Conversion and Management 52(2011) 781-788, 2011.

[12]. R. Bettochi, M. Pinelli, P.R. Spina, M. Venturini, and M. Burgio, "Set up of a robust neural network for gas turbine simulation," ASME Turbo Expo 2004, Vienna, Austria. 
[13]. R. Bettochi, M. Pinelli, P.R. Spina, and M. Venturini, “Artificial intelligent for the diagnostics of gas turbine, Part 1: Neural network approach,” ASME Turbo Expo 2005, Nevada, USA.

[14]. M. Basso, L. Giarre, S. Groppi, and G. Zappa, "NARX models of an industrial power plant gas turbine", IEEE Transactions on Control Systems Technology, 2004.

[15]. Y. Yoru, T.H. Karakoc, and A. Hepbasli, "Application of artificial neural network (ANN) method to exergetic analyses of gas turbines," International Symposium on Heat Transfer in Gas Turbine Systems, Antalya, Turkey, 2009.

[16]. S. Simani, and R. Patton, "Fault diagnosis of an industrial gas turbine prototype using a system identification approach,” Control Engineering Practice 16(2008) 769-786, 2008.

[17]. M. Fast, M. Assadi, and S. De, "Condition based maintenance of gas turbines using simulation data and artificial neural network: A demonstration of feasibility," ASME Turbo Expo 2008, Berlin, Germany.

[18]. M. Fast, M. Assadi, and S. De, "Development and multi-utility of an ANN model for an industrial gas turbine,” Journal Of Applied Energy, Vol. 86, Issue 1, pp. 9-17, January 2009.

[19]. M. Fast, T. Palme, and M. Genrup, "A novel approach for gas turbines monitoring combining CUSUM technique and artificial neural network," ASME Turbo Expo 2009, Orlando, Florida, USA.

[20]. M. Fast, T. Palme, and A. Karlsson, "Gas turbines sensor validation through classification with artificial neural networks," ECOS 2009, Brazil.

[21]. M. Fast, and T. Palme, "Application of artificial neural network to the condition monitoring and diagnosis of a combined heat and power plant,” Journal Of Energy, Vol. 35, Issue 2, pp. 114-1120, February 2010.

[22]. M. Fast, "Artificial neural networks for gas turbine monitoring," Doctoral Thesis, Division of Thermal Power Engineering, Department of Energy Sciences, Faculty of Engineering, Lund University, Sweden, 2010.

[23]. P.R. Spina, and M. Venturini, "Gas turbine modelling by using neural networks trained on field operating data," ECOS 2007, Padova, Italy.

[24]. S.O.T. Ogagi, R. Singh, and S.D. Probert, "Multiple-sensor faultdiagnosis for a 2-shaft stationary gas turbine,” Applied Energy 71 (2002) 321-339.

[25]. J. Arriagada, M. Genrup, A. Loberg, and M. Assadi, "Fault diagnosis system for an industrial gas turbine by means of neural networks," Proceedings of the International Gas Turbine Congress 2003, Tokyo, Japan.

[26]. M.R.B Tavakoli, B. Vahidi, and W. Gawlik, "An educational guide to extract the parameters of heavy-duty gas turbines model in dynamic studies based on operational data”, IEEE Transactions on Power Systems, Vol. 24, No. 3, August 2009.

[27]. G. Cybenko, "Approximation by Superpositions of a Sigmoidal Function," Mathematics of Control, Signals, and Systems, Vol. 2, pp. 303-314, 1989.

\section{BIOGRAPHIES}
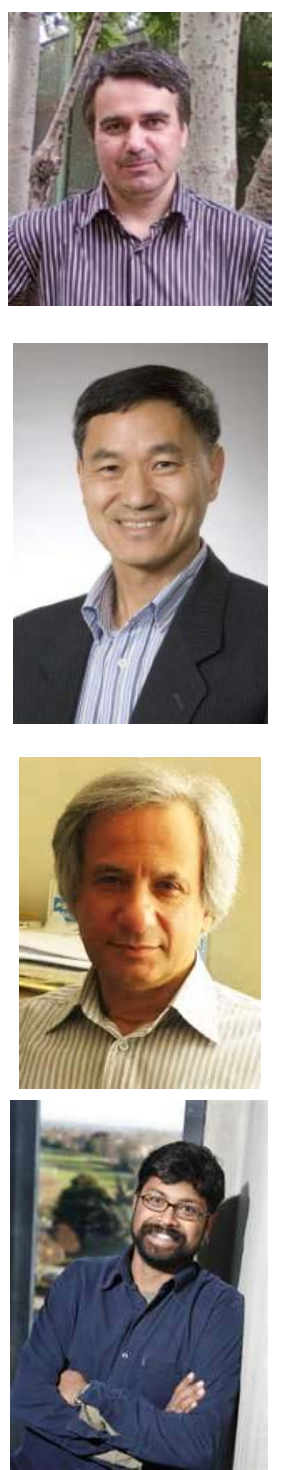

Hamid Asgari is a PhD Candidate in Department of Mechanical Engineering at University of Canterbury (UC), Christchurch, New Zealand.

He has worked as a chief engineer in different industrial companies for more than 14 years.

Mr. Asgari is a member of Iranian Society of Mechanical Engineers (ISME), American Society of Mechanical Engineers (ASME), and Iranian Society of Instrument and Control Engineers (ISICE).

XiaoQi Chen has received his $\mathrm{PhD}$ from Department of Electrical Engineering \& Electronics, The University of Liverpool, UK.

He is a Professor in Department of Mechanical Engineering at University of Canterbury (UC), Christchurch, New Zealand.

Professor Chen has extensively worked with the companies through collaborative research projects. His research interests are mobile and assistive robotics, manufacturing systems and automation, tissue engineering and energy harvesting.

Mohammad Bagher Menhaj received his $\mathrm{PhD}$ from school of electrical and computer engineering at Oklahoma State University (OSU), USA.

He is a Professor in Department of Electrical Engineering at Amir Kabir University of Technology (AUT), Tehran, Iran.

Professor Menhaj research interests are adaptive control systems, computational intelligence, neural networks, fuzzy logic, and genetic computations.

Raazesh Sainudiin received his $\mathrm{PhD}$. in Statistics from Cornell University, New York, USA.

$\mathrm{He}$ is a senior lecturer in Department of Mathematics and Statistics at University of Canterbury (UC), Christchurch, New Zealand.

Dr. Sainudiin research interests include statistical inference of stochastic processes embedded within stochastically evolving networks, such as statistical decision problems in population genetics, phylogenetics, ecological genetics, airtraffic management and set-valued statistics. 